\title{
An Adaptive Triple-Path Mechanism for Congestion Control in Computer Networks
}

\author{
Jassim M. Abdul-Jabbar* \\ $*$ drjssm@yahoo.com
Computer Engineering Department, College of Engineering, University of Mosul, Mosul, Iraq. \\ Omar A. Hazim
}

\begin{abstract}
It is known that congestion may widely appear in computer networks which apply open shortest path first (OSPF) protocol. The use of such protocol may lead to serve congestion states in some network links while another links are either not used or underutilized. In this paper, an adaptive triple-path congestion control routing mechanism is proposed. This mechanism is based on adaptive adding up to two new additional paths between source and destination nodes, in addition to original shortest path when there is a possibility of congestion in the network links. These two new paths use some information about the congestion and loading states gathered from a network predictor called wavenet-based link status predictor (WBLSP). The first additional path uses a routing algorithm called wavenet-based congested link scaling (WBCLS), while the second uses another algorithm called wavenet-based loaded link-aware with congested link scaling (WBLLACLS). The proposed congestion control routing approach is tested and its performance is evaluated using OPNET 14.5 Modeler. The results are compared with the results of applying traditional OSPF routing protocol on two different tested network topologies and similar loading scenarios.

Keywords-Computer network; Congestion control; Link load; Link utilization; OSPF; Prediction; Wavelet neural networks (wavenet).

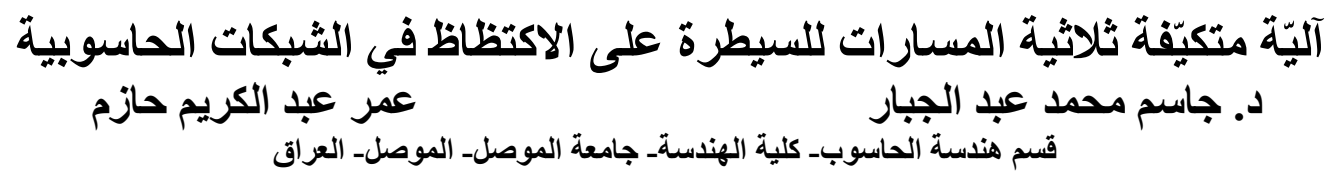

الخلاصة

إنه من المعروف أن الاكتظاظ يظهر بشكل واسع في شبكات الحاسوب التي تطبق بروتوكول توجيه فتح المسار الأقصر

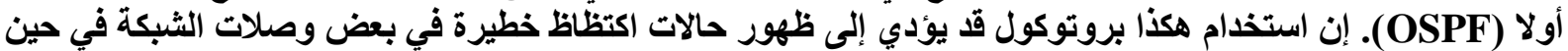

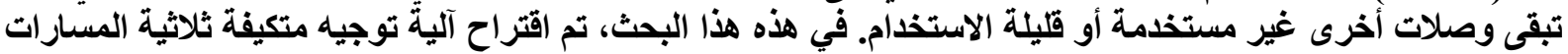

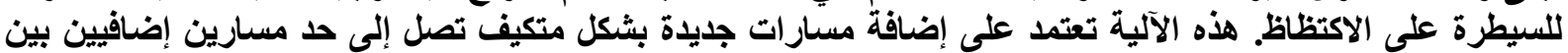

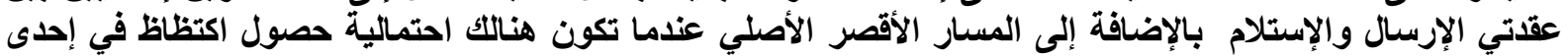

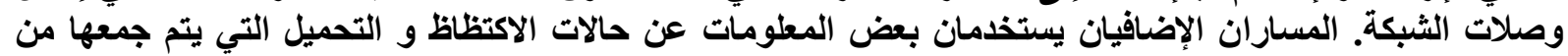

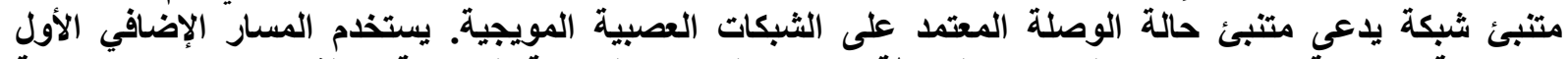

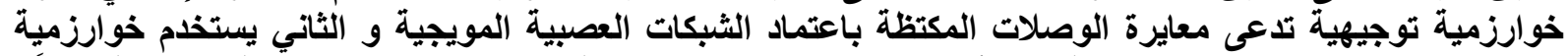

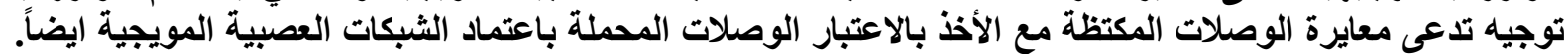

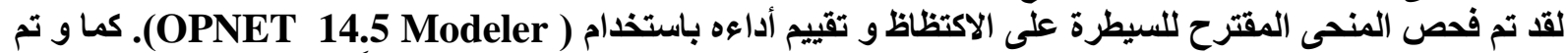

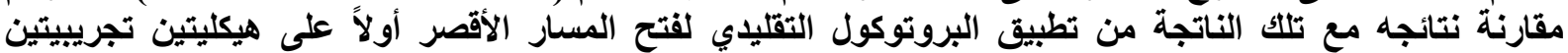
مختلفتين و بنفس سيناريوهات التحميل.
\end{abstract}

Received: 19 - 5 - 2013

Accepted: 7 - 10 - 2013 

Al-Rafidain Engineering
Vol.22
No. 4
May 2014

\section{Introduction}

As the computer networks based applications are increased, the required bandwidths are also increased. Each network is composed of some physical links that have finite bandwidths and user nodes. Many links are shared among multiple independent users. As the case with any finite resources, network may be congested when the load demand exceeds network capacity [1], [2].

Network routing, which is the selection of the path along which the packets are travelling from source node to destination node [3], the selected path has a strong impact on the transmission and network performance. Current path selection strategies, like open shortest path first (OSPF), which is used inside autonomous systems (AS), only depend on network topology and do not consider the traffic pattern between the nodes, because it is based on shortest path defined by a suitable fixed metric. The use of the shortest path only may lead to many situations in which the links on shortest path routes become congested while others are not used or with low use [4].

Firstly, it was believed that the amount of adaption assigned to OSPF, can react to congestion. Unfortunately, congested links often remain undetected because of the way OSPF assesses link connectivity. If a link flaps constantly due to congestion, but at least 1 out of every 4 Hello messages is received, OSPF does not detect the problem. If the congestion is severe and no Hello messages are received from a neighbor, it is automatically considered down because OSPF makes no distinction between hardware failures and congestion. Thus, the involved router will not be further used and all the traffic will be rerouted to a different link which in turn can also become congested. Consequently the solution adopted by OSPF does not resolve the underlying problem that of transmitting too much traffic on a single link [5].

In the present Internet, congestion control mechanisms rely on queue management algorithms (dropping packets randomly or based on their priority) or TCP (Transmission Control Protocol) congestion avoidance (by reducing the sending rate). From the end-user perspective, these solutions are not optimal because they either result in some loss of packets or reduced bit rate operations, there by affecting the quality of transmission. Therefore, some of new researches propose techniques for solving congestion by changing the paths of traffic to avoid congested links or by adding new paths [5].

This paper proposes the use of wavenet neural network [6], [3] which shows in recent researches a good prediction ability to predict the congestion and loading states of the network links in the next time interval. Such information states are used to add new adaptive paths between source and destination nodes, while some other techniques are provided to avoid network instability due to bouncing in predictor outputs in some cases.

The rest of this paper is organized as follows: Section 2 briefly presents previous related works regarding using network parameters prediction in congestion control algorithms. Section 3 contains wavenet fundamentals. The used wavenet predictor and how it can be interfaced with the applied congestion controlling algorithm are illustrated in section 4 . Section 5 describes the proposed adaptive triple-path routing mechanism. The simulation tools and testing networks topologies are described in section 6 . The simulation results are displayed and discussed in section 7. Finally, section 8 gives some conclusions. 


\section{Related Works}

Recently, several researchers tried to solve congestion problems by integrating network parameters prediction techniques with a single or multiple path routing. In [7] C. Chang proposed a congestion control for integrated voice and data direct sequence Code division multiple access (CDMA) cellular system based on Fuzzy/neural by providing adequate access probability for users. The controller was designed to contain three parts: recurrent neural network, interference predictor as a fuzzy performance indicator, and access probability generator based on predicted next step interference sample and performance indicator. A simple feed forward neural network is proposed by J. Bivens and B. Szymanski in [8] to predict severe congestion in a network and the source or sources responsible for the congestion. They also designed a simple control method for limiting the rate of the offending sources so that congestion can be avoided. H. Wang and M. Ito suggested in [9] a new routing algorithm called load sensitive adaptive routing (LSAR), in which link weights were updated online according to link loads. The suggested algorithm tries to drive mapping function between link utilization and link weight (cost). LSAR gave better performance than OSPF/ IS-IS in term of packet drop rate in many cases but in some cases packet drop rate was increases. The author of [10] designed a routing technique based on constraint-based routing (CBR) that combines the strength of prediction with an innovative link-state cost. CBR is applied in circuit-switched networks and it reduces the impact of routing inaccuracy on the blocking probability. Uncongested shortest path first (USPF) algorithm was proposed in [11] to solve congestion problem in an open shortest path first (OSPF) based on best effort network. A previously trained feed-forward neural network was used to predict the congestion problem in the computer network links and then a control method was applied to select the shortest paths, while excluding the most congested links.

Recently, a sample of using neural network predictor with multiple-path routing was accomplished by the authors of [12] when they designed an intelligent routing framework, consisting of a situation-aware multipath routing algorithm and a routing management system involving neural networks-based predictors with multi-task learning. The solution is characterized by QoS-awareness, load balancing and self-management. Also they proved their concept by simulation and a practical implementation of predictive QoS-aware multipath routing in a real test environment.

More recently, A. Jasim [3] used a wavelet neural network for congestion prediction and congestion-load state predictions using previous link utilization values and he displayed in his work several solutions that belong to single- and dual-path routing strategies to solve links congestion or at least minimize its negative effects on the network performance.

In this paper, an adaptive triple-path mechanism is proposed for congestion prediction and control. Two of those paths are wavenet-based designed to adaptively serve besides the traditional OSPF one.

\section{Wavenet Fundamentals}

Wavenet can be considered as a particular case of the feed forward basis function neural network model [6], [12]. In ordinary network, several types of bases functions, such as radial basis functions, splines and polynomial functions of synapse neurons are used instead of sigmoidal function. The connection weights are taken to represent the corresponding coefficients. The output layer performs the sum of the output of all synapse neurons. Since wavelets have shown their excellent performance in non-stationary signal analysis and non- 
linear function modeling, then it can be easily proved that neural networks with wavelet basis function, or wavenets, can provide higher availability of rate of convergence for the approximation problem than any ordinary feed forward neural network. The structure of WNN is shown in Figure 1. The approximated output signal of the network of Figure 1 can be expressed as follows [13]:

$$
y_{i}=f\left[\sum_{k=1}^{K} w_{k i} \sum_{m=1}^{M} x_{m}(t) h_{m}\left[\left(x-b_{k}\right) / a_{k}\right]\right]
$$

where $x_{m}(m=1,2, \ldots, M)$ is the input for the $m^{\text {th }}$ training vector $X(t)$, $y_{i}(i=1,2, \ldots, N)$ is the output for the $i^{\text {th }}$ training vector $Y(t), M$ is number of nodes of the input layer, $\mathrm{K}$ is number of nodes of hidden layer, $w_{k i}$ is the weight between the $k^{\text {th }}$ hidden layer neuron and the $i^{\text {th }}$ output layer node, $h(x)$ is wavelet mother function $a_{k}$ and $b_{k}$ are the scaling and translating parameters and $f$ is the nonlinear function.

The Mexican-Hat function is used in this paper as a wavelet basis function that is given the following function:

$$
h(u)=\left(1-u^{2}\right) e^{\frac{-u^{2}}{2}}
$$

The applied training algorithm is the gradient that is commonly used to minimize the error and obtain the suitable network parameters. The process can be summarized as that the variations of error energy function with respect to each network parameter i.e., the gradient factors are calculated. Then these factors are used as incremental factors to update the current network parameters in the direction leads to minimize the error [3].

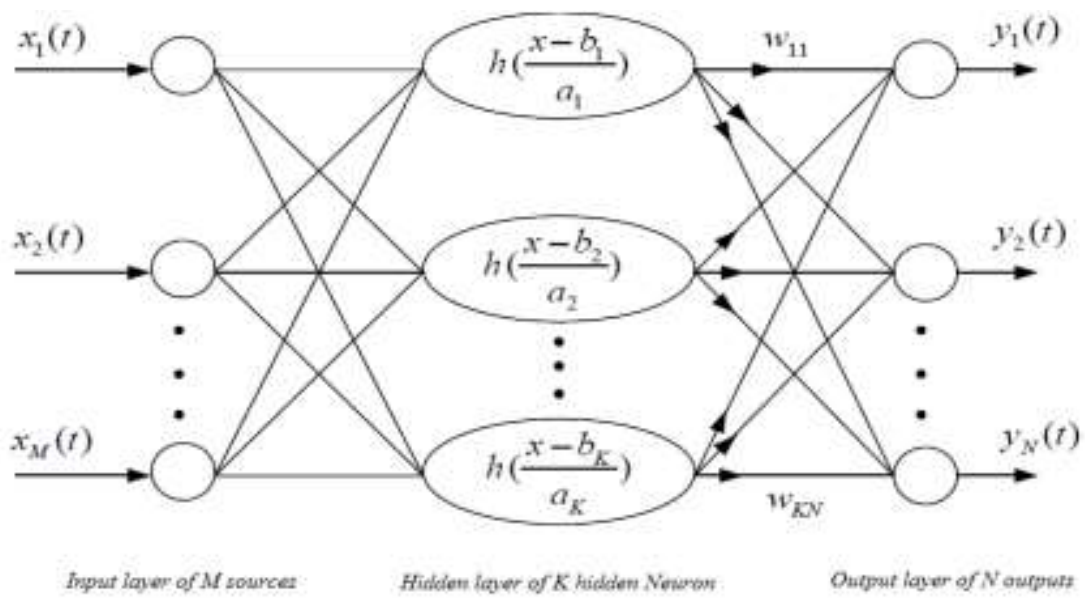

Figure 1 WNN structure.

\section{The Adopted Wavenet-Based Link Status Predictor (WBLSP)}

An independent WNN network is suggested for each unidirectional link in the computer network, all of such WNNs operate in parallel to compute the output prediction vectors. The structure of single WNN predictor is shown in Figure 2. Each WNN predictor consists of 3 nodes in the input layer, 5 nodes (wavelons) in the hidden layer and 2 nodes in the output layer. Each input is the square of the average link utilization for one minute in the near past and can be given by 


$$
U_{i}=\left[\begin{array}{lll}
u_{1}^{2} & u_{2}^{2} & u_{3}^{2}
\end{array}\right]
$$

where $u_{i}$ represents the average of utilization values of $i$ th previous minute.

Accordingly, the total inputs of the proposed WNN reflect the utilization behavior during the last three minutes. The Mexican-hat wavelet is used as an activation function in the hidden layer nodes since it is very appropriative for function approximation and prediction because it is continuous, differentiable, provide a softer output, and improve the interpolation capabilities. It also reduces the number of iterations resulting in a faster convergence and a good escaping from local minima [3]. It should be noted that the use of square functions distribute the inputs in a more efficient manner, decreases the miss error rates and make the size of the WNN predictor smaller.

Each WNN generates two bit-string outputs representing both congestion and load states of the corresponding link. In the first output, the congestion bit is set when one of the average of utilization values of the last three minutes is greater than $70 \%$ or the average of utilization values of current minute may be greater than $70 \%$ else the output is cleared. And the loading bit is set when one of the averages of utilization values of the last three minutes is greater than $40 \%$ or the average of utilization values of current minute may be greater than $40 \%$. The total predicted state is described by two vectors the congestion-state vector and load-state vector. If a computer network consists of $\mathrm{k}$ links, there will be $\mathrm{k}$ bits in each vector.

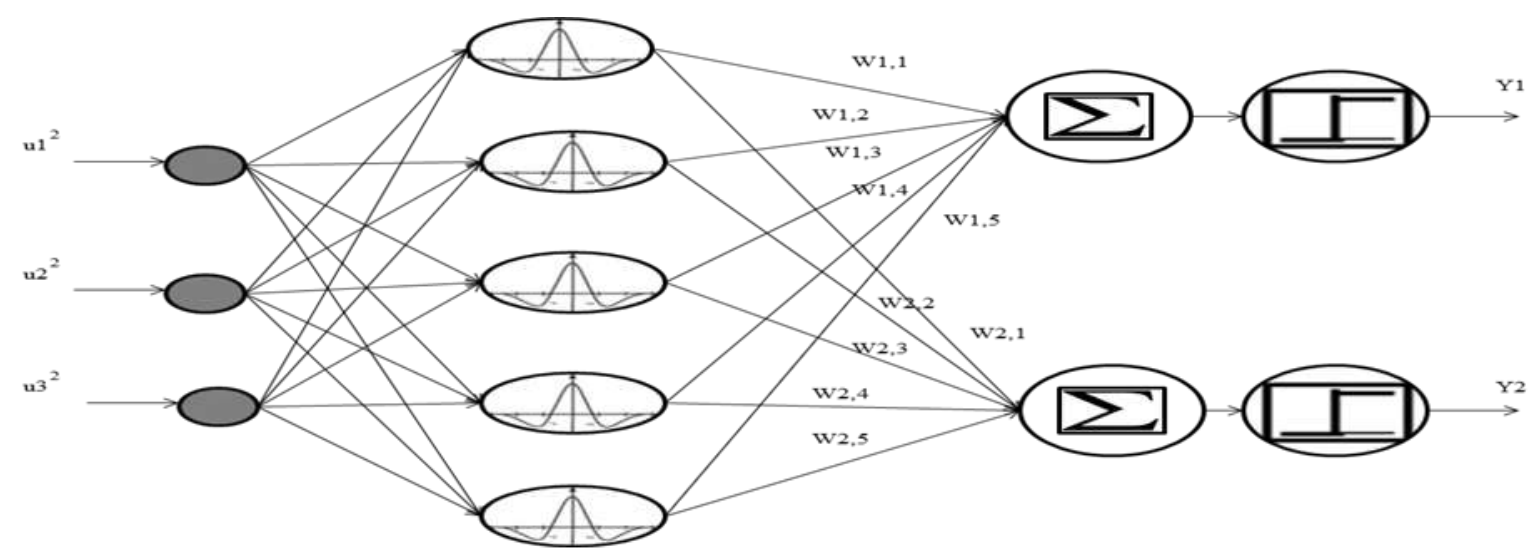

Figure 2 The structure of the WNN for WBLSP.

Finally, the outputs of the predictor (Y1,Y2), that represent congestion and loading prediction respectively, are not directly interfaced to network congestion control algorithm because it may cause instability in network behavior due to bouncing in predictor outputs when the network load in a specific network link or links bounce up and down the congestion threshold (70\%). Figure 3 shows the interfacing circuit that is used to interface the predictor outputs to congestion control algorithm. The interfacing circuit ensures the links that are processed by a congestion controller still assumed congested until the link become underuse. The output of the interfacing circuit is computed as follows:

$C^{+}=Y_{1}+C^{-} \cdot Y_{2}$ 


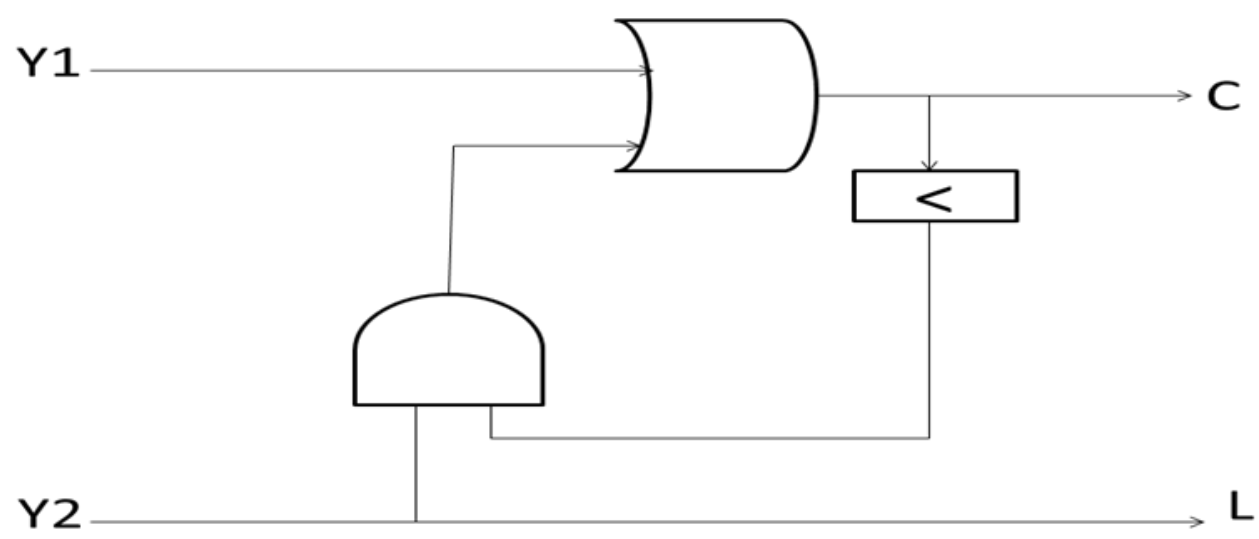

Figure 3 Predictor outputs interfacing circuit

\section{The Proposed Adaptive Triple-Path Congestion Control Mechanism}

The proposed congestion control routing mechanism is developed by extending the traditional OSPF routing protocol from single path routing to a triple-path routing. The additional paths use the information gathered from the predictors in addition to original links weights to determine the second and third routes and to distribute loads over these three paths. Routing table is modified to include three entries per destination as shown in Table 1.

Table 1 Triple-path routing table sample

\begin{tabular}{|c|c|c|c|c|c|c|c|c|c|c|c|c|}
\hline Destination Network & \multicolumn{3}{|c|}{ N1 } & \multicolumn{3}{|c|}{ N2 } & \multicolumn{3}{c|}{ N3 } & \multicolumn{3}{c|}{ N4 } \\
\hline Cost & 5 & 5 & 5 & 9 & 11 & 19 & 12 & 14 & 14 & 4 & 6 & 18 \\
\hline Next Router & A & A & A & E & A & D & F & B & B & C & D & E \\
\hline
\end{tabular}

For a given destination, there are three entries each contains next router field and cost field. Distribution of loads on these three routes is arranged that the first route serves $70 \%$ of the total load and the rest is distributed evenly on the other routes. The cause of choosing this distribution scheme is that in the case of congestion prediction in network link, congested link load will be decreased by $30 \%$ so that there is a chance to recover congestion by lightening link load, at the same time, tries to avoid a new congestion problem in the second path links (uncongested shortest path) that are originally having their own load from other paths which may be the first path (shortest path). These three routing fields are updated according to the mechanism is shown in Figure 4, where P1_enable and P2_enable are just flags used to control the updating of the second and third entries of routing tables.

At first, all routing entries for each destination are initialized by shortest path and the whole load flow over a single shortest path like OSPF and stay on this situation until the congestion predictor predicts that there is one or more network links may become congested in the next time interval. Any change in congestion state can cause a change in the congestion vector bits states. When the first change in congestion vector is detected the network enters a dual-path routing phase by using wavenet-based congested link scaling (WBCLS) algorithm 
to update the second and third entries. WBCLS uses the following function to compute links weights before using Dijkstra's algorithm on them to compute the shortest path:

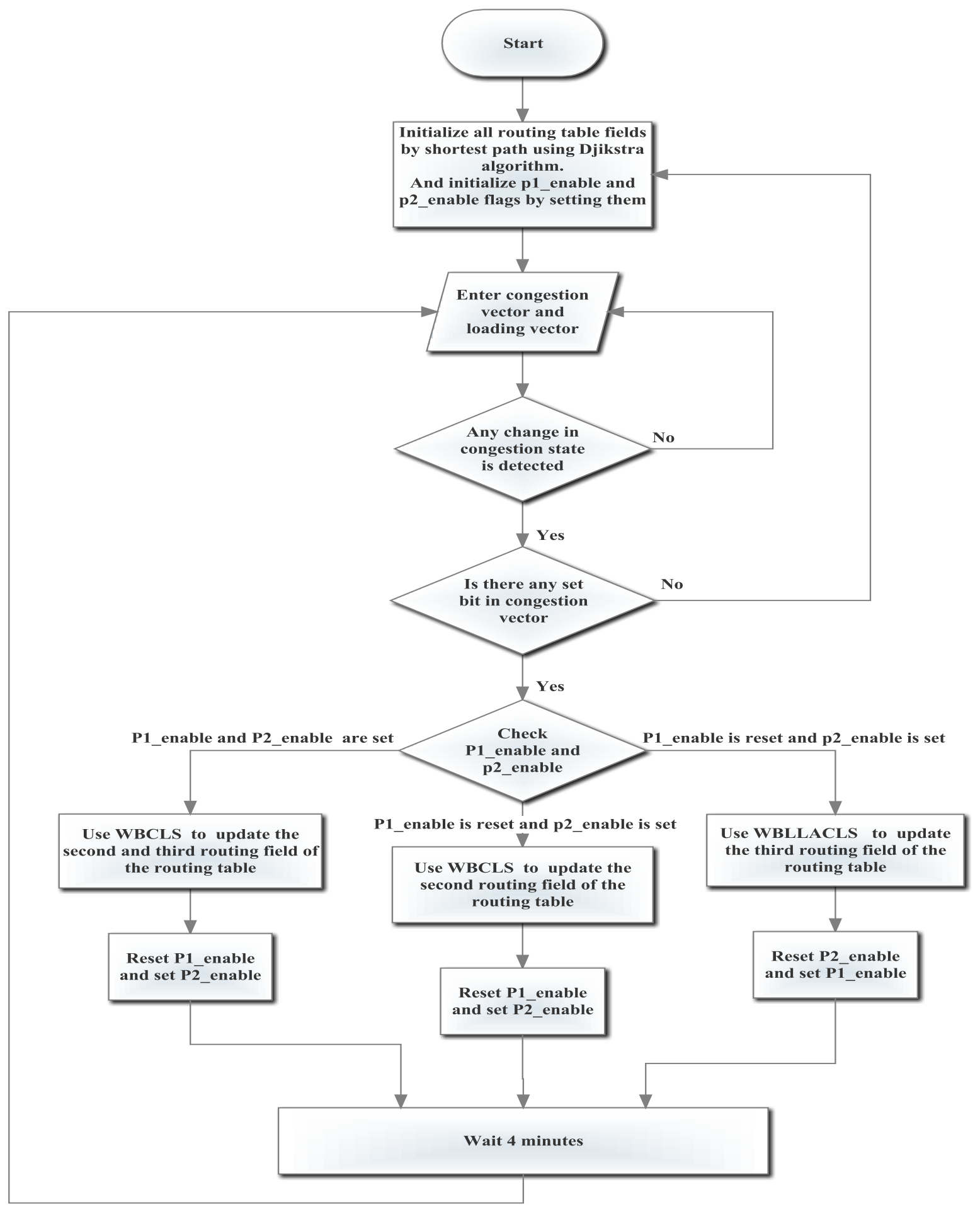

Figure 4 Adaptive triple-path algorithm 
where:

NW (i): the new weight of the ith link.

$\mathrm{W}$ (i): the original weight of the ith link.

$\mathrm{C}$ (i): the ith bit of congestion vector that corresponding to the ith link.

The WBCLS add 100 to the weight of each congested link so when applying Dijkstra's algorithm congested link will be avoided and the path with least congested links will be chosen. In this phase the second and third routing table entries have the same values for the next router field and cost field but they may differ from the first entries for each distention node and therefore $70 \%$ of load is passed across OSPF shortest path and 30\% of load is sent along the second WBCLS path. During such phase, a network is still in dual-path routing as long as no new changes in network congestion state are detected. When a new change is detected in congestion state and that change indicates there is congestion in a new link, or some but not all congested links are recovered, the triple-path routing phase is turned on. The triple-path phase start by using wavenet-based loaded link-aware with congested link scaling (WBLLACLS) to update only the third routing entry of each destination and keep the two other without any modification until a new change in congestion prediction is predicted. So the third entry may contain different route from the second one for the same destination. WBLLACLS use the following function to compute links cost before applying Dijkstra's algorithm:

$N W(i)=W(i)+C(i) * 90+L(i) * 10$

where:

NW (i): the new weight of the ith link.

W (i): the original weight of the ith link.

$\mathrm{C}$ (i): the ith bit of congestion vector that corresponding to the ith link.

L (i): the ith bit of loading vector that corresponding to the ith link.

WBLLACLS is like WBCLS in adding 100 to weights of congested links to be avoided but it takes the advantage of loading prediction to eschew the links used by the first two routes by adding 10 to its original cost values. In this time there are three paths for each destination and each one may differ from the two other. In this time, load distribution is as follows: $70 \%$ of total load pass throw the OSPF path, $15 \%$ of total go along second WBCLS path and the rest $15 \%$ of the load are served by the third WBLLACLS. This is not the end, if a new change is detected in congestion state, the algorithm updates only the second entry by using WBCLS algorithm and then when additional changes are detected in congestion vector the third-path are updated by WBLLACLS and the algorithm stays on this sequential updating process until all the changes in congestion vector indicate there are no possibility for congestion in any one of the network links. At that time, the algorithm reinitializes all three entries by the shortest path and the network returns to single routing phase. To enhance the stability of the algorithm and prevent any unexpected behavior of the network due to hazard in predictors output after each update in routing tables the mechanism waits 4 minutes before it returns to use predictor's outputs. The cause of choosing this time particularly is that none of those predictor's inputs have hazard time. At the end, it must be mentioned that the three 
routes are also changed due to topology changes and links failure like traditional OSPF protocol.

\section{Computer Network Topologies and Scenarios}

Two different network topologies are used. Each can be viewed as a single autonomous system (AS) with single autonomous boundary router or as a single area with one area border router and each one represents a campus or university network. Figures 5 show these two topologies (Topology 1 and Topology 2, respectively). The two networks are simulated using OPNET 14.5 simulator.

To generate load demand and for the purpose of accuracy, three network application (HTTP, FTP and EMAIL) are used in the simulation in four different scenarios. The load scenarios descriptions are presented in Table 2.

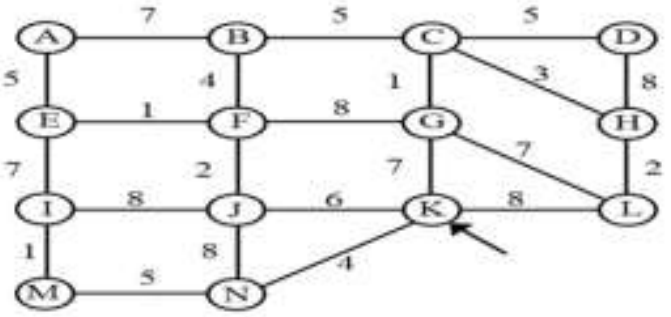

(a) Network topology 1

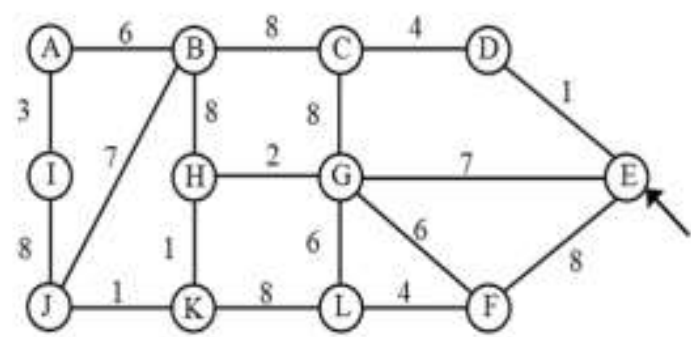

Figure 5 Network topologies

Table 2 Network load scenarios

\begin{tabular}{|l|l|}
\hline Load Style & Description \\
\hline Scenario 1 & $\begin{array}{l}\text { Load is applied throw several edge routers while others are unloaded (only } \\
\text { light load are applied on the other edge routers). }\end{array}$ \\
\hline Scenario 2 & $\begin{array}{l}\text { All edge routers have load that is fluctuated in a manner so all links } \\
\text { connected to the border router are congested but not simultaneously. }\end{array}$ \\
\hline Scenario 3 & $\begin{array}{l}\text { All edge routers apply same high loading scheme so that many links } \\
\text { connected to the border router are congested simultaneously. }\end{array}$ \\
\hline Scenario 4 & $\begin{array}{l}\text { All edge routers apply high loading so that all links connected to the border } \\
\text { router are congested simultaneously }\end{array}$ \\
\hline
\end{tabular}

All these scenarios run for 6 hours first by using OSPF routing protocol on both topologies and then by using adaptive triple-path mechanism.

\section{Simulation Results and Discussion}

As mentioned before, the two topologies are used to test the two routing strategies OSPF and adaptive triple-path routing. Page response time are collected and compared for all scenarios on both topologies, in Figures 6 and 7, as a one of global network performance indicators. Link utilization and link queuing delay are collected as a link performance indicators statistics. For proposed adaptive triple-path mechanism, the number of changes in routing tables is collected as indicator for suitability of the proposed algorithm for practical use. Because there are no changes in network topology and no failures occurred in network elements, there are no changes in routing tables after the completion of them (when the 
traditional OSPF protocol continuous running). Table 3 and 4 compares between the performances of both routing strategies by illustrating the top five values for the averages of queuing delay times. While summaries of links utilization are displayed in Tables 5 and 6 for topology 1 and 2 respectively, attached with number of changes in routing table due to changes of congestion states in the two network topologies, which will be used to evaluate the stability of the adaptive triple-path routing.

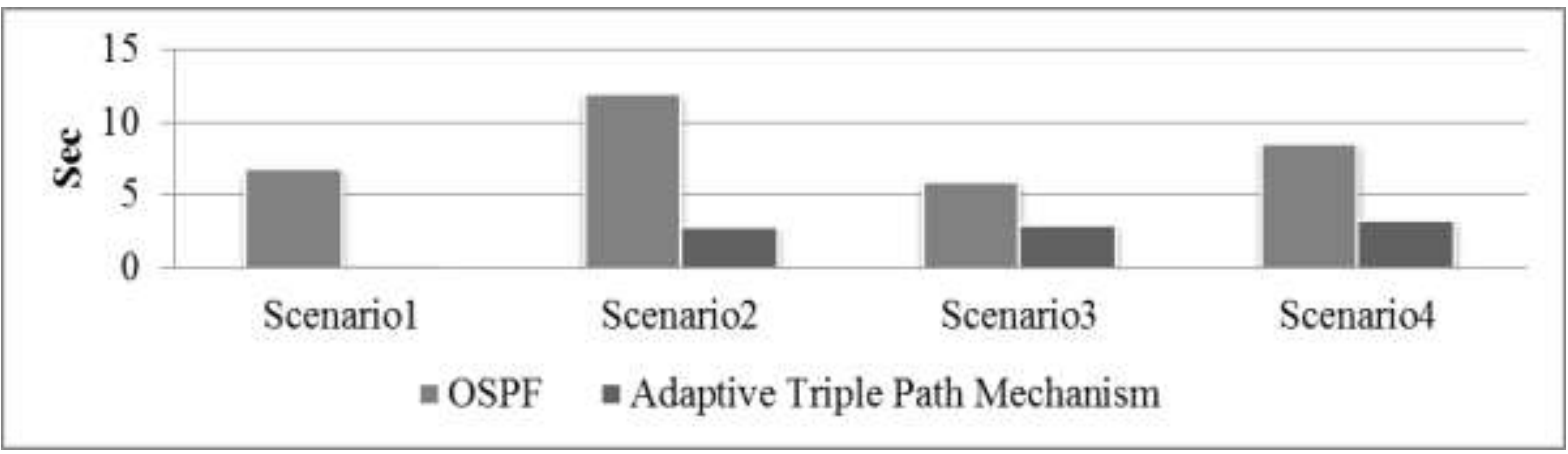

Figure 6 HTTP page response time comparison for Topology 1

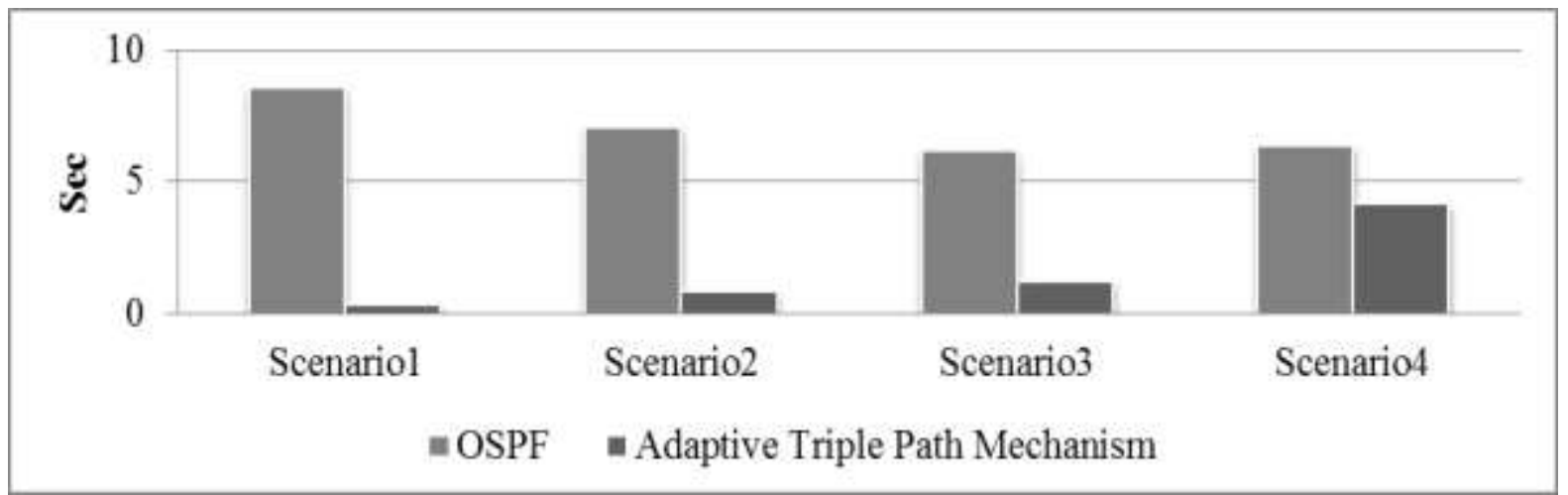

Figure 7 HTTP page response time comparison for Topology 2

Figures 6 and 7 clearly show how can the use of adaptive triple-path routing brilliantly succeeds in minimizing the page response times for all scenarios and topologies from that for OSPF. That because the proposed adaptive triple-path routing mechanism utilizes more possible links to access the packets destination than OSPF which uses only the links that belongs to the shortest paths. The adaptive triple-path routing also succeeds in decreasing the top levels for averages of queuing delays values of Scenarios 1, 2 and 3, as shown in Table 3 and 4, for both topologies but in Scenario 4, for both topologies the decrease in link queuing delays for some congested links is accompanied with the increase of those delays for some other links. This increase in Scenario 4 comes from the fact that the applied load exceeds the capacity of the network and the work of OSPF accordingly is almost optimal. This means that adding more paths from sources to destinations in such scenario will not resolve the congestion problem. But the triple-path mechanism does its best and what is gained exceeds what is lost. Consequently, the triple-path mechanism has smaller page response time than OSPF. Table 5 and 6 indicate, specially the variance and standard deviation columns that have smaller values for triple-path mechanism than OSPF, that means that the proposed mechanism 
distribute the load over network links in more efficient manner for all scenarios and both topologies. Also in Table 5 and 6, the number of routing tables' changes column record reasonable numbers of routing tables updates for all scenarios and for both topologies which indicates that the use of predictor's output interfacing circuits and the interchangeable approach in exchanging the responsibility of updating the routing tables have succeeded in preventing the routing oscillation.

\section{Conclusions}

An adaptive triple-path mechanism has been proposed for solving congestion problem in computer networks. The proposed mechanism has been simulated and its performance has been evaluated and compared with the performance of traditional OSPF protocol for two different topologies with several loading scenarios. The performance results show the superiority of the proposed mechanism on the traditional OSPF in all scenarios from the point of view of global performance and in most of these scenarios from the point of view of individual links performance in which the applied loads do not exceed the network capacity. It has also been shown that the adaptive triple-path mechanism is a stable adaptive routing algorithm since it appears with small number of changes in routing table. From all of that it can be argued that the proposed mechanism can achieve greater network resources utilization and increase network capacity without disturbing the network stability.

Table 3 Top 5 averages queuing delay values for topology 1

\begin{tabular}{|c|c|c|c|c|c|c|c|c|c|c|c|c|}
\hline & \multicolumn{6}{|c|}{ OSPF } & \multicolumn{6}{|c|}{ Adaptive triple-path mechanism } \\
\hline \multirow{2}{*}{ Scenario 1} & Links & KJ & KG & CG & FJ & KL & Links & KG & KJ & KL & KN & LH \\
\hline & Values & 1.923 & 0.93 & 0.019 & 0.015 & 0.014 & Values & 0.031 & 0.02 & 0.016 & 0.014 & 0.01 \\
\hline \multirow{2}{*}{ Scenario 2} & Links & KG & KL & KJ & KN & LK & Links & KG & KL & KJ & LK & KN \\
\hline & Values & 1.206 & 1.189 & 1.17 & 1.169 & 0.032 & Values & 0.725 & 0.548 & 0.38 & 0.165 & 0.058 \\
\hline \multirow{2}{*}{ Scenario 3} & Links & KN & KJ & KL & KG & NK & Links & KG & KJ & KN & KL & NK \\
\hline & Values & 1.716 & 1.658 & 0.035 & 0.032 & 0.016 & Values & 0.099 & 0.062 & 0.056 & 0.039 & 0.013 \\
\hline \multirow{2}{*}{ Scenario 4} & Links & KN & KJ & KL & KG & FJ & Links & KG & KN & KL & KJ & LK \\
\hline & Values & 1.956 & 1.943 & 1.018 & 0.931 & 0.025 & Values & 3.751 & 0.142 & 0.127 & 0.112 & 0.078 \\
\hline
\end{tabular}

Table 4 Top 5 averages queuing delay values for topology 1

\begin{tabular}{|c|c|c|c|c|c|c|c|c|c|c|c|c|}
\hline & \multicolumn{6}{|c|}{ OSPF } & \multicolumn{6}{|c|}{ Adaptive triple-path mechanism } \\
\hline \multirow{2}{*}{ Scenario 1} & Links & KJ & JK & KL & KH & IL & Links & KJ & KL & JK & KH & IL \\
\hline & Values & 1.966 & 0.026 & 0.019 & 0.01 & 0.01 & Values & 0.089 & 0.016 & 0.014 & 0.011 & 0.009 \\
\hline \multirow{2}{*}{ Scenario 2} & Links & KH & KJ & KL & FE & JK & Links & KH & HE & LK & KJ & KL \\
\hline & Values & 1.121 & 1.117 & 0.02 & 0.018 & 0.011 & Values & 0.179 & 0.079 & 0.076 & 0.031 & 0.019 \\
\hline \multirow{2}{*}{ Scenario 3} & Links & KJ & KH & JK & KL & $\mathbf{F E}$ & Links & KH & $\mathbf{K J}$ & KL & JK & $\mathrm{HE}$ \\
\hline & Values & 1.996 & 0.198 & 0.034 & 0.015 & 0.015 & Values & 0.336 & 0.298 & 0.018 & 0.014 & 0.009 \\
\hline \multirow{2}{*}{ Scenario 4} & Links & KJ & KH & KL & IL & JK & Links & KH & HK & KJ & LI & JK \\
\hline & Values & 1.951 & 1.437 & 0.246 & 0.022 & 0.022 & Values & 4.451 & 0.239 & 0.16 & 0.125 & 0.095 \\
\hline
\end{tabular}


Table 5 Utilization results summaries for topology 1

\begin{tabular}{|c|c|c|c|c|c|c|}
\hline Scenarios & Routing algorithm & $\begin{array}{c}\text { Maximum } \\
\text { utilization } \\
\%\end{array}$ & $\begin{array}{c}\text { Average } \\
\text { utilization } \\
\%\end{array}$ & $\begin{array}{c}\text { Variance of } \\
\text { utilization }\end{array}$ & $\begin{array}{c}\text { Standard } \\
\text { deviation } \\
\text { of } \\
\text { utilization }\end{array}$ & $\begin{array}{c}\text { Number } \\
\text { of changes } \\
\text { in routing } \\
\text { table }\end{array}$ \\
\hline \multirow[b]{2}{*}{ Scenario 1} & OSPF & 68.7 & 16.44 & 505.53 & 22.48 & ----- \\
\hline & $\begin{array}{l}\text { Adaptive triple- } \\
\text { path mechanism }\end{array}$ & 49.44 & 14.54 & 190.44 & 13.8 & 3 \\
\hline \multirow[b]{2}{*}{ Scenario 2} & OSPF & 44 & 15.54 & 252.41 & 15.89 & ----- \\
\hline & $\begin{array}{l}\text { Adaptive triple- } \\
\text { path mechanism }\end{array}$ & 44.92 & 18.42 & 184.1 & 13.57 & 12 \\
\hline \multirow[b]{2}{*}{ Scenario 3} & OSPF & 68.88 & 20.66 & 470.18 & 21.68 & ---- \\
\hline & $\begin{array}{l}\text { Adaptive triple- } \\
\text { path mechanism }\end{array}$ & 57.53 & 20.23 & 310.7 & 17.63 & 6 \\
\hline \multirow[b]{2}{*}{ Scenario 4} & OSPF & 68.59 & 23.81 & 601.28 & 24.52 & ----- \\
\hline & $\begin{array}{l}\text { Adaptive triple- } \\
\text { path mechanism }\end{array}$ & 68.16 & 23.58 & 440.68 & 20.99 & 11 \\
\hline
\end{tabular}

Table 6 Utilization results summaries for topology 2

\begin{tabular}{|c|c|c|c|c|c|c|}
\hline Scenarios & Routing Algorithm & $\begin{array}{c}\text { Maximum } \\
\text { utilization } \\
\%\end{array}$ & $\begin{array}{c}\text { Average } \\
\text { utilization } \\
\%\end{array}$ & $\begin{array}{l}\text { Variance of } \\
\text { utilization }\end{array}$ & $\begin{array}{c}\text { Standard } \\
\text { deviation } \\
\text { of } \\
\text { utilization }\end{array}$ & $\begin{array}{l}\text { Number of } \\
\text { changes in } \\
\text { routing } \\
\text { table }\end{array}$ \\
\hline \multirow[b]{2}{*}{ Scenario 1} & OSPF & 68.47 & 12.39 & 304.29 & 17.44 & ----- \\
\hline & $\begin{array}{l}\text { Adaptive triple- } \\
\text { path mechanism }\end{array}$ & 62.62 & 13.79 & 225.36 & 15.01 & 4 \\
\hline \multirow[b]{2}{*}{ Scenario 2} & OSPF & 43.1 & 15.7 & 235.72 & 15.35 & ----- \\
\hline & $\begin{array}{l}\text { Adaptive triple- } \\
\text { path mechanism }\end{array}$ & 41.53 & 16.25 & 183.31 & 13.54 & 13 \\
\hline \multirow[b]{2}{*}{ Scenario 3} & OSPF & 64.13 & 19.57 & 419.59 & 20.48 & ----- \\
\hline & $\begin{array}{l}\text { Adaptive triple- } \\
\text { path mechanism }\end{array}$ & 62.25 & 20.56 & 297.23 & 17.24 & 7 \\
\hline \multirow[b]{2}{*}{ Scenario 4} & OSPF & 62.6 & 24.04 & 550.41 & 23.46 & $\begin{array}{ll}----- \\
\end{array}$ \\
\hline & $\begin{array}{l}\text { Adaptive triple- } \\
\text { path mechanism }\end{array}$ & 62.53 & 22.62 & 350.62 & 18.72 & 8 \\
\hline
\end{tabular}

\section{References}

[1] G. Boanea, M. Barabas, A. B. Rus, and V. Dobrota, "Design Principles and Practical Implementation of a Situation Aware Multipath Routing Algorithm", Intern. Conf. on Software, Telecommunications and Computer Networks 2010, Split-Bol, Croatia, 2010, pp. 321-325.

[2] E. Soedarmadji, "Generalized Network Routing Metrics and Algorithms", Ph.D. thesis, California Institute of Technology, 2008.

[3] A. Jasim "Wavenet-Based Computer Network Routing with Congestion Control", Ph.D. Thesis in Electrical Engineering, Basrah University, 2012.

[4] J. Moy, "OSPF Anatomy of an Internet Routing Protocol”, Addison-Wesley, 1998.

[5] M. Barabas, G. Boanea and V. Dobrota "Multipath Routing Management using Neural NetworksBased Traffic Prediction", The Third International Conference on Emerging Network Intelligence, 20-25 Nov., 2011, pp. 118-124. 
[6] J. Abdul-Jabbar, M. Alwan and A. Jasim "A New Wavenet-Based Network Congestion Predictor WBCP", International Conference on Future Communication Networks, 9-12 April 2012, pp.12-17.

[7] C. Chang et. al, " Fuzzy/Neural Congestion Control for Integrated Voice and Data DSCDMA/FRMA Cellular Networks", IEEE Journal on Selected Area in Communications, Vol. 18, No. 2, 2000, pp. 283-293.

[8] J. Bivens, M. Embrechts and B. Szymanski, "Network Congestion Arbitration and Source Problem Prediction using Neural Networks", Smart Engineering System Design Journal, Vol. 4, 2002, pp. 243-252

[9] H. Wang and M. Ito, "Dynamics of Load-Sensitive Adaptive Routing", IEEE conference on Communication ICC, Vol. 1, 2005, pp. 213-217.

[10] X. Masip-Bruin, E. Marin-Tordera, M. Yannuzzi, R. Serral-Gracia, and S. Sanchez-Lopez, "Reducing the Effects of Routing Inaccuracy by Means of Prediction and an Innovative LinkState Cost", IEEE Communications Letters, Vol. 14, No. 5, 2010, pp. 492-494.

[11] Z. N. Abdulkader, "Path Finding with Reduced Congestion in Computer Networks using Artificial Neural Network", M. Sc. Thesis in Computers and Mathematic Sciences, University of Mosul, 2010.

[12] M. Barabas, G. Boanea and V. Dobrota "Multipath Routing Management using Neural NetworksBased Traffic Prediction", The Third International Conference on Emerging Network Intelligence, 20-25 Nov., 2011, pp. 118-124.

[13] J. Abdul-Jabbar, "Multi-Basis Wavenet-Based Stator Resistance Identification in DCT Induction Motor System", Al-Rafidain Engineering Journal, Vol. 18, No. 2, 2010, pp. 1-14.

[14] D. Veitch , "Wavelet Neural Networks and Their Application in The Dynamical Systems", M.Sc. Thesis in Data Analysis, Networks and Nonlinear Dynamics, Department of Mathematics, University of York, 2005.

[15] C. Lin, "Wavelet Neural Networks with a Hybrid Learning Approach", Journal of Information Science and Engineering, Vol. 22, 2006, pp. 1367-1387. 